\title{
Uso y Recursos Tecnológicos de los Entornos Personales de Aprendizaje con Estudiantes de los Grados de Maestro en Educación Infantil y Primaria
}

\author{
Ana M. Ortiz-Colón ${ }^{(1) \star}$, José L. Maroto ${ }^{(2)}$ y Miriam Agreda Montoro ${ }^{(1)}$ \\ (1) Universidad de Jaén, Dpto. de Pedagogía. Campus Las Lagunillas, s/n. 23071. Jaén - España. \\ (2) I.E.S. Oretania. Linares. Consejería de Educación. Junta de Andalucía - España. \\ Email: aortiz@ujaen.es; jlmaroto@iesoretania.es; magreda@ujaen.es
}

* Autora a quien debe ser dirigida la correspondencia

Recibido Abr. 13, 2017; Aceptado Jun. 19, 2017; Versión final Jul. 13, 2017, Publicado Oct. 2017

\begin{abstract}
Resumen
El artículo presenta los resultados más relevantes de la innovación desarrollada con estudiantes universitarios de los Títulos de Grado de Educación Infantil y Educación Primaria, en referencia a su entorno personal de aprendizaje, PLE. El objetivo general de la investigación es analizar los PLE de cada estudiante en el foro de la plataforma ILIAS, y conocer el uso que hacen de los recursos tecnológicos utilizados. La experiencia se ha llevado a cabo con una muestra de 225 estudiantes de la Universidad de Jaén (España), siguiendo una metodología de corte descriptivo, El análisis de datos se realiza a través de un proceso de codificación apoyado por el programa Aquad 7, que aporta cierto grado de cuantificación de los códigos analizados. Los resultados del estudio muestran la coincidencia de determinados recursos tecnológicos en todos los estudiantes por un lado y por otro en el uso que le dan en sus prácticas.
\end{abstract}

Palabras clave: entorno personal de aprendizaje; educación superior; foro; plataforma virtual; estudiantes universitarios; recursos tecnológicos

\section{Use and Technological Resources of Personal Learning Environments with Students of the Degree in Preschool and Primary Education}

This paper aims to present the most relevant results of innovation developed with undergraduate students of the Master's Degree in Preschool and Primary Education, in reference to their Personal Learning Environment, PLE. The main goal of the study was to analyse the PLE of each student in the forum of the ILIAS platform, and to know the use of the technological resources used. The research was carried out with a sample of 225 students from the University of Jaén (Spain). The data analysis is performed through a coding process with a categorical system supported by the Aquad 7 program, which provides some degree of quantification of the analysed codes. On the one hand, the results of the study show coincidence of certain technological resources in all the students and on the use they give them in their practices, on the other hand.

Keywords: personal learning environments; higher education; forum; virtual platforms; university students; technological resources 


\section{INTRODUCCIÓN}

Las experiencias más recientes en torno al uso de internet indican un cambio drástico en la educación, sólo similar a lo que fue la aparición de la imprenta en su momento. Hablar en el momento presente de cómo las prácticas educativas se han modificado tras la irrupción de internet es hoy una realidad que no admite discusión en todos los contextos educativos, no sólo universitarios. En el ámbito educativo, poco a poco han ido haciéndose hueco, tratando de demostrar que el aprendizaje puede llegar a ser más efectivo con el uso de las TIC siempre y cuando, la actitud hacia las mismas sea receptiva y abierta (Marín, et al., 2016). De hecho, es extraño encontrar en el momento actual instituciones de educación superior que no dispongan de un campus virtual para sus usuarios.

Si hacemos una revisión de las fuentes más comunes y los medios de comunicación al uso, podemos ver la evolución en los nuevos entornos basados en internet y en las herramientas de las web 2.0 (Couros, 2011), y cómo y dónde podemos aprender en un contexto globalizado. Así pues, la aparición del concepto de Entorno Personal de Aprendizaje, PLE, por sus siglas en inglés -Personal Learning Environment-, está teniendo un amplio desarrollo en la comunidad tanto científica como en la práctica, en contextos académicos y educativos en general, apareciendo diferentes enfoques y perspectivas de los mismos.

Existen claras diferencias sobre lo que se entiende por PLE, en base a los diferentes contextos de estudio (Adell y Castañeda, 2010; Attwell, 2007; Downes, 2007; Van Harmelen, 2008; Casquero, 2013). Si bien, siendo diversas las definiciones que se han ofrecido de los PLE, en líneas generales pueden agruparse en torno a dos grandes tendencias, unas de carácter pedagógicas/educativas y las otras de carácter tecnológicas/instrumentales. En referencia a la tendencia pedagógica/educativa o psicopedagógica (Cabero, Barroso y Llorente, 2010), los PLE se consideran sistemas que vienen a ayudar a los estudiantes y a los docentes en la gestión de su propio aprendizaje. Se trata de un proceso de apoyo al aprendizaje para lograr los objetivos en cuanto a fijar metas, formalizar contenidos y comunicarse con los demás. Desde esta perspectiva el concepto de PLE incluye la integración de los elementos de la formación tanto formal como informal con el apoyo y uso de redes sociales y otros recursos y sistemas conectados personalmente. En definitiva, el PLE se entiende como la forma en que los individuos usan la tecnología para su aprendizaje en el contexto actual de la sociedad del conocimiento, dando respuesta a las nuevas formas de conocimiento que se están generando.

Por otro lado, dentro de la tendencia de carácter tecnológico/instrumental, se encuentra el conjunto de herramientas de aprendizaje, servicios, entornos y recursos que pueden utilizar los estudiantes. Fiedler y Pata (2009) los definen como una colección de instrumentos, materiales y recursos humanos que una persona conoce, y tiene acceso en el contexto de un proyecto educativo en un momento dado. Estos autores aunque no obvian un proyecto educativo, le dan más importancia al aspecto tecnológico. Para Attwell (2008), un PLE está formado por un conjunto de herramientas que nos permiten en los espacios analógicos, leer, reflexionar y compartir (Adell y Castañeda, 2010). Son escasas las investigaciones cuyo foco de atención está en los usos que realizan los estudiantes en estos entornos (Castañeda y Adell, 2014; Attwell et al., 2013; Chaves et al., 2015, 2016; Coll, et al., 2014; Castañeda y Soto, 2010; Llorente, 2013; Valtonen et al., 2012), planteándose la necesidad de ir más allá de las herramientas, hacia actividades y procesos que configuran el PLE.

En este trabajo, se consideran los entornos personales de aprendizaje como el conjunto de materiales, aplicaciones y servicios digitales y recursos humanos que una persona utiliza para aprender en los diferentes contextos educativos y profesionales en los que participa. Se plantea como objetivo, analizar los PLE de cada estudiante en el foro de la plataforma ILIAS, y conocer el uso que hacen de los recursos tecnológicos utilizados los estudiantes de los Grados de Educación Infantil y Educación Primaria de la Universidad de Jaén, considerando como base del estudio el foro en la plataforma virtual como herramienta asíncrona de comunicación en el campus virtual de las instituciones de educación superior.

El objetivo general de la investigación pretende analizar los PLE de cada estudiante en el foro de la plataforma ILIAS, y conocer el uso que hacen de los recursos tecnológicos utilizados.

\section{METODOLOGÍA}

La metodología seguida en el trabajo es de corte descriptivo (Cerda et al., 2017), aportando datos cuantitativos con objeto de conocer y analizar el uso que los estudiantes hacen de las tecnologías digitales como herramientas que componen su PLE. Para ello se analiza el contenido de los foros mediante el programa Aquad 7, que a su vez, nos ofrece la posibilidad de cierto grado de cuantificación, proporcionando frecuencias absolutas (FA) y porcentajes de frecuencias absolutas (\%FA) de los códigos observados. 
El estudio se llevó a cabo durante el curso 2015-2016 con estudiantes de a la Facultad de Humanidades y Ciencias de la Educación de la Universidad de Jaén (España), de los Grados de Maestro en Educación Infantil, en dos grupos distintos y el Grado de Maestro en Educación Primaria en un solo grupo. Los sujetos participantes fueron por un lado, 81 estudiantes que pertenecían al Grupo A (GAl) y 62 estudiantes pertenecientes al Grupo B del Grado de Maestro en Educación Infantil (GBI) (identificados así para el estudio) y por otro, 80 estudiantes del Grupo A del Grado de Maestro en Educación Primaria (GAP) (identificado de este modo, igualmente para el estudio). Del análisis de datos del foro, se extrae la distribución de los estudiantes que participaron definitivamente por titulación, grupo y sexo, tal y como se aprecia en la tabla 1, por lo que para la selección de la muestra se ha empleado un muestreo no probabilístico intencional y se han utilizado grupos naturales.

Tabla 1: Distribución de estudiantes por titulación, grupo y sexo

\begin{tabular}{|c|c|c|c|c|c|c|}
\hline & & Hombres & Mujeres & Total Hombres & Total Mujeres & Total \\
\hline \multirow{2}{*}{ Ed. Infantil } & Grupo A (GA!) & 7 & 74 & \multirow{2}{*}{13} & 130 & 143 \\
\cline { 2 - 7 } & Grupo B (GBI) & 6 & 56 & & & \\
\hline \multirow{2}{*}{ Ed. Primaria } & Grupo A (GAP) & 37 & 43 & 37 & 43 & 80 \\
\hline
\end{tabular}

\section{Procedimiento}

Mediante una selección intencional de sujetos, se propuso a los estudiantes a través del foro de la Plataforma llias de la Universidad de Jaén, que respondieran a los siguientes interrogantes ¿Qué herramientas y estrategias componían su PLE? Y que reflexionaran sobre el uso que le daban a dichas herramientas en su PLE. La plataforma que la Universidad de Jaén utiliza en su campus virtual es ILIAS disponible como software libre. Entre sus ventajas ofrece herramientas de comunicación síncronas y asíncronas. La herramienta que se ha utilizado en el trabajo, ha sido el foro ya que al ser los mensajes públicos, sirve para plantear temas de interés general entre todo el grupo, siendo moderados por el profesorado.

Una vez recogidos y registrados los datos que nos permitían obtener información acerca del objeto de estudio, y analizar qué herramientas de la web 2.0 usaban los estudiantes, se observó la frecuencia con la que aparecía cada respuesta y éstas fueron agrupadas en categorías de contenido. Para ello se ha seguido la clasificación realizada por Attwell (2008) y Adell y Castañeda (2010), que establecen tres dimensiones: herramientas de acceso a la información, herramientas de creación y edición de información y herramientas de relación con otros. A su vez, dentro de estas dimensiones se ajustaron las categorías establecidas por Attwell (2008) y Adell y Castañeda (2010), en nuestro estudio. En la primera dimensión, se analizaron, sitios de publicación, repositorios y bases de datos, video, multimedia, objetos de aprendizaje, lectores de RSS, sitios de noticias y Plataformas. En la segunda, en referencia a las herramientas de creación y edición de información, las categorías fueron, creación de Wikis, sitios ofimáticos de escritorio y en red, herramientas de mapas mentales y herramientas de edición. En la dimensión de herramientas de relación con otros, las categorías fueron, herramientas de red social, herramientas de las que emerge una red social, relación basada en la comunicación, y establecimiento de relaciones personales (Castañeda y Gutiérrez, 2010).

Una vez establecidas las categorías, se codificaron para su análisis con el programa Aquad 7, estudiando porcentajes de los estudiantes y de los grupos. Para conocer la concordancia de los codificadores que en nuestro estudio fueron tres, se aplicó el índice Kappa de Fleiss, obteniendo un $\mathrm{K}=0,77$, por lo que estamos según los autores (Fleiss,1981 y Altman,1991), ante un índice bueno o muy bueno, por lo que se garantiza que las codificaciones se ajustan a la realidad objeto de estudio. El análisis se completa con algunas tablas e imágenes de los resultados del estudio, así como datos extraídos del foro, fruto del análisis de contenido realizado, lo que nos va a permitir extraer conclusiones acerca del uso de las herramientas del PLE de los estudiantes del estudio, analizando los segmentos de contenido de las aportaciones al foro (Feliz, 2012; Huber, 2003).

\section{RESULTADOS Y DISCUSIÓN}

El análisis de los datos, se presenta en base a las dimensiones, categorías y códigos extraídos del estudio (Attwell, 2008; y Adell y Castañeda, 2010). En primer lugar presentamos las frecuencias y porcentajes de las categorías y subcategorías mediante los códigos asignados en la tabla 2, pudiéndose observar que dentro de la dimensión herramientas de acceso a la información, los estudiantes usan exclusivamente el repositorio de la plataforma llias. En cuanto a los objetos de aprendizaje, no mencionan el uso de ninguno 
de ellos, ni el uso de sitios de noticias, manifiestan conocer el uso de Adobe acrobat para la lectura de noticias.

Las herramientas de acceso a la información hacen referencia a los sitios de publicación, bases de datos, objetos de aprendizaje, repositorios institucionales, portales de información específica, entre otros. El estudio nos ha permitido conocer el uso que hacen los estudiantes de los tres grupos de estas herramientas. El Grupo A de Educación Infantil (GAI), es el que usa en mayor porcentaje los Blog (BLOG), tanto Wordpress como Blogger en un 91,4\%, N=74 a diferencia del Grupo B de Educación Infantil (GBI) con un 80,6\% siendo N=50 y el Grupo A de Educación Primaria (GAP), en un 38,8\% y N=31. En el uso de Wikis con N=42, la proporción es de un $51,9 \%$ en el GAl, siendo de un $71 \%$ en el GBI con $\mathrm{N}=44$ y un $40 \%$ en el GAP con $\mathrm{N}=32$. En referencia al uso que hacen de la Plataforma llias (ILIA), es el GBI el que realiza un uso más elevado, siendo $\mathrm{N}=62$ y un porcentaje de 100 , seguido del grupo GAI con un $79,0 \%$ y $N=64$ y en tercer lugar el GAP en un $42,5 \%$ y $\mathrm{N}=34$.

Destaca del análisis de contenido del foro, la entrada de una de las participantes en cuanto al uso que hace de la plataforma Ilias [GAI_ 00054] (...) En cuanto a las herramientas y estrategias de lectura, de las cuales, obtengo la información que busco, entre ellas se encuentran: llias, que es la que más uso puesto que es ahí donde todos los profesores nos comparten información; Google, donde busco información que veo conveniente y Youtube (YOUT), en esta aplicación, busco información en vídeos que muestren lo que quiero aprender pues me resulta más fácil de esta manera.

Tabla 2: Herramientas de acceso a la información

\begin{tabular}{|l|c|c|c|c|c|c|c|}
\hline Categorías & Códigos & GAI & GBI & GAP & $\% G A I$ & $\% G B I$ & $\% G A P$ \\
\hline 1.1. Blog, (Wordpress, Blogger) & BLOG & 74 & 50 & 31 & 91,4 & 80,6 & 38,8 \\
\hline 1.2. Wikis (Wikipedia) & WIKI & 42 & 44 & 32 & 51,9 & 71 & 40,0 \\
\hline 1.3. Ilias & ILIA & 64 & 62 & 34 & 79,0 & 100 & 42,5 \\
\hline 1.4. Youtube, & YOUT & 75 & 60 & 34 & 93,8 & 96,8 & 42,5 \\
\hline 1.5. Vimeo, & VIME & 12 & 34 & 30 & 14,8 & 54,8 & 37,5 \\
\hline 1.6. Slideshare (presentaciones) & SLID & 12 & 20 & 34 & 14,8 & 32,3 & 42,5 \\
\hline 1.7. Repositorios,... & REPO & 40 & 30 & 30 & 49,4 & 48,4 & 37,5 \\
\hline 1.8. Google reader & GOOG & 74 & 45 & 34 & 91,4 & 72,6 & 42,5 \\
\hline 1.9. Adobe acrobat & ADOB & 40 & 42 & 32 & 49,4 & 67,7 & 40,0 \\
\hline
\end{tabular}

En cuanto a Youtube (YOUT), alcanza en el GBI un porcentaje del 96,8\%, $\mathrm{N}=60$, seguido del GAI de un $93,8 \%, N=76$ y del GAP con un porcentaje del $42,5 \%, N=34$. El uso de Youtube, lo expresaba la alumna en la entrada al foro [GBI_00011] (...) También uso con frecuencia Youtube (YOUT), ya que me da la oportunidad de acceso a Videos (VIME) educativos que me sirven como eficaces recursos formativos $y$ educativos. En cuanto al uso de Vimeo (VIME), se mantiene en igual proporción que el anterior, con porcentajes del 54,8\%, N=34 en el GBI, seguidos del 37,5\%, N=30 en el GAP y de 14,8\%, N=12 en el GAl.

El uso de Slideshare (SLID) y Presentaciones por los estudiantes, N=34, obtiene en el GAP un 42,5\%, seguido de un $32,3 \%, \mathrm{~N}=20$ en el $\mathrm{GBI}$ y de un 14,8\%, N=12 en el GAl. En referencia al uso de Repositorios (REPO), se observa que el mayor porcentaje lo alcanza el GAl con un porcentaje del 49,4\%, N=40, seguido del $\mathrm{GBI}$ con un $48,4 \%$ y $\mathrm{N}=30$ y de un $37,5 \%, \mathrm{~N}=30$, en el GAP. La categoría Google reader (GOOG) es utilizado por los tres grupos en porcentajes que oscilan desde el 91,4\% (N=74) en el GAl, 72,6\% (N=45) en el $\mathrm{GBI}$ y $42,5 \%(\mathrm{~N}=34)$ en el GAP, tal y como quedó recogido en el mensaje [GAl_003] (...) También me es de mucha ayuda Google dónde busco información y accedo a la página web online de la biblioteca de la universidad para ver si tienen libros que me interesan. La herramienta Adobe acrobat (ADOB) es utilizada en un $67,7 \%(N=42)$ en el $G B I$, seguido por el $G A I$ en un $49,4 \%(N=40)$ y del GAP en un $40 \%(N=32)$. 


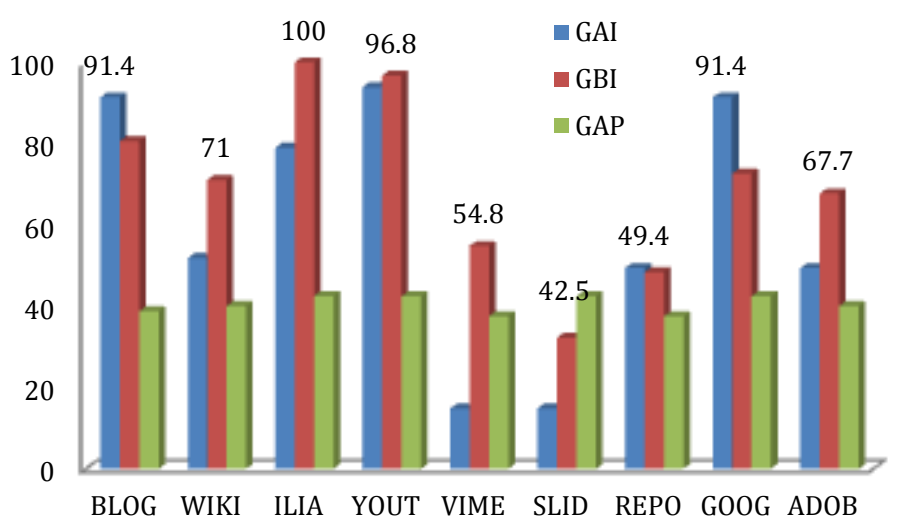

Fig. 1: Dimensión 1. Herramientas de acceso a la información

Los resultados obtenidos en la dimensión (Herramientas de acceso a la información), tal y como podemos observar en la figura 1, nos indican que el porcentaje más elevado respecto a las categorías 1.1. (BLOG), 1.7 (REPO) y 1.8 (GOOG), lo obtiene el GAl; el GBI obtiene los mayores porcentajes en las categorías 1.2 (WIKI), 1.3 (ILIA), 1.4 (YOUT), 1.5 (VIME) y 1.9 (ADOB); el GAP, obtiene la mayor puntuación en la categoría 1.6 (SLID). La segunda dimensión que establecen Attwell (2008) y Adell y Castañeda (2010), hace referencia a las herramientas de creación y edición de información.

Tabla 3: Herramientas de creación y edición de información

\begin{tabular}{lccccccc}
\hline Categorías & Códigos & GAI & GBI & GAP & $\% G A I$ & $\% G B I$ & $\% G A P$ \\
\hline 2.1. Creación de Wikis & CREW & 40 & 50 & 34 & 49,38 & 80,65 & 42,50 \\
2.2. Office (M.Word) & OFFI & 71 & 62 & 44 & 87,65 & 100 & 55,00 \\
2.3. Video & VIDE & 24 & 30 & 34 & 29,63 & 48,39 & 42,50 \\
2.4 Dibujo (Paint, pinterest,..) & PAIN & 48 & 40 & 34 & 59,26 & 64,52 & 42,50 \\
2.5. Presentaciones (M. Power Point) & PRES & 48 & 50 & 44 & 59,26 & 80,65 & 55,00 \\
\hline
\end{tabular}

Las herramientas de creación y edición de información analizadas, se refieren a creación de Wikis, Uso de office, Video, Dibujo y Presentaciones, el total de códigos referidos a creación de Wikis (CREW), es de $80,65 \%, \mathrm{~N}=50$ en el $\mathrm{GBI}$, seguido del $\mathrm{GAI}$ con un $49,30 \%, \mathrm{~N}=40$ y un $42,5 \%$ en el GAP con $\mathrm{N}=34$. Dentro de la categoría 2.2. office (OFFI), observamos que el $100 \%$ de los estudiantes del GBI lo usan, ( $N=62$ ), seguidos del $87,65 \%$, $(\mathrm{N}=71)$ y del $55 \%(\mathrm{~N}=44)$, tal y como queda recogido por diferentes estudiantes en el análisis del foro [GBI_00047] (...) Uso también Microsoft Office (OFFI), que es lo que más utilizo para trabajos de la universidad. [GAl_0008](...) Éstas son, las que me permiten transformar la información que anteriormente he recogido. Las que más utilizo son Microsoft Word (OFFI) y Power Point (PRES). A parte de éstas, también utilizo Wordpress (BLOG) y Movie Maker (VIDE). [GAP_00060](...) Para guardar y compartir información suelo utilizar Microsoft Word (OFFI), Microsoft Power Point (PRES) y Excel (OFFI), entre otros. Considero destacable también el uso de Google drive (DRIV), el cuál considero muy útil ya que a la hora de trabajar en equipo facilita mucho más esa tarea, permitiendo que podamos editar dos personas o más a la vez en línea e ir modificando el documento y que se guarde en la nube.

En cuanto a la categoría 2.3. Video (VIDE), obtiene puntuaciones que oscilan del $48,39 \%$ a $42,50 \%$ y $29,63 \%$ de los grupos GBI, GAP y GAI con N=30, 34 y 24 respectivamente. La categoría 2.4. Dibujo (PAIN), obtuvo un porcentaje de respuestas en el GBI de $64,52 \%$ con $\mathrm{N}=40$, seguido del GAl con un $59,26 \%, \mathrm{~N}=48$ y un $42,50 \%$ el GAP con $\mathrm{N}=34$. La última categoría de la dimensión, Presentaciones (PRE), obtiene un $80,65 \%, \mathrm{~N}=50$ en el GBI, seguidas del GAI $(59,26 \%)$ y $\mathrm{N}=48$ y del GAP (55\%9 con $\mathrm{N}=44$. Los resultados de la dimensión 2, véase fig. 2, obtiene los mayores porcentajes en el GB de Educación Infantil, en todas las categorías. En cuanto a la tercera dimensión referida a herramientas de relación con otros, los resultados obtenidos en los tres grupos, pueden observarse en la tabla 4. 


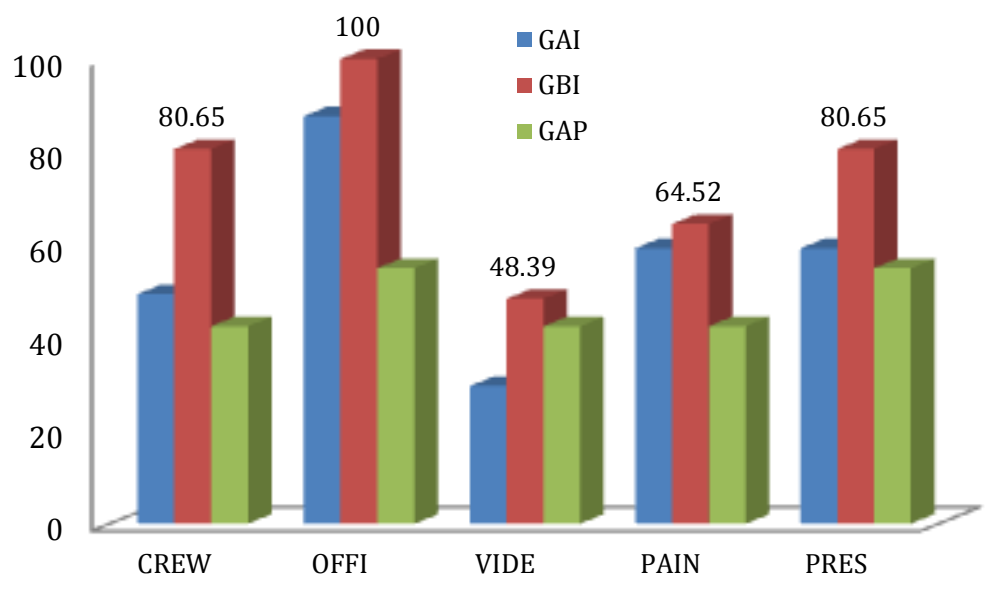

Fig. 2: Dimensión 2. Herramientas de creación y edición de información

En relación a la categoría 3.1. Whatssap (WHAT) observamos que las puntuaciones más altas las obtiene el GBI, $96,8 \%$ con $\mathrm{N}=60$, seguido del $\mathrm{GAl}(92,59 \%)$ y $\mathrm{N}=75$ y un $50 \%$ el GAP con $\mathrm{N}=40$, expresadas en las diferentes entradas al foro por los estudiantes [GBI_00082] (...) ¿qué me decías de Whatsapp (WHAT)? sin duda un recurso muy socorrido para cuando tienes una duda que puedes preguntarle a un compañero y que no puede esperar. [GAl_00073](...) Whatsapp (WHAT), en la que comparto información relevante; Gmail (OUTL), en la cual, entre compañeras nos mandamos información importante y que vemos conveniente $y$ Google Drive (DRIV), donde nos guardamos documentos importantes en línea para archivarla de manera segura y consultarla cuando sea necesario. [GAP_00013](...)También comparto información que considero relevante en mis Redes sociales y se los envío vía chat a mis contactos, mediante Whatsapp (WHAT) y el correo electrónico de Gmail (OFFI). Youtube (YOUT), es la categoría más utilizada en el GAl con una puntuación del $93,8 \%, \mathrm{~N}=76$, seguida del $\mathrm{GBI}$, con un porcentaje del $80,65 \%, \mathrm{~N}=50$ y el GAP con un $50 \%$ con $\mathrm{N}=40$. Google Drive (DRIV) obtiene una frecuencia del $67,7 \%, \mathrm{~N}=42$ en el $\mathrm{GBI}$, seguida del $59,26 \%$ y $\mathrm{N}=48$ en el GAl y de un $50 \%, \mathrm{~N}=40$ en el GAP, observándose los mismos resultados que en la categoría Slideshare (SLID).

Tabla 4: Herramientas de relación con otros

\begin{tabular}{lccccccc}
\hline Categorías & Códigos & GAI & GBI & GAP & $\% \mathrm{GAI}$ & $\% \mathrm{GBI}$ & $\% \mathrm{GAP}$ \\
\hline 3.1. Whatsapp & WHAT & 75 & 60 & 40 & 92,59 & 96,8 & 50,00 \\
3.2. Youtube, & YOUT & 75 & 50 & 40 & 93,8 & 80,65 & 50,00 \\
3.3. Slideshare & SLID & 48 & 40 & 40 & 59,26 & 64,5 & 50,00 \\
3.4. Google drive & DRIV & 48 & 42 & 40 & 59,26 & 67,7 & 50,00 \\
3.5. Twiter & TWIT & 74 & 56 & 38 & 91,4 & 90,32 & 47,50 \\
3.6. Outlook (gmail, hotmail) & OUTL & 68 & 50 & 48 & 84 & 80,65 & 60,00 \\
3.7. Facebook & FACE & 70 & 55 & 44 & 86,42 & 88,7 & 55,00 \\
3.8. Skype & SKYP & 42 & 45 & 32 & 51,85 & 72,6 & 40,00 \\
3.9. Instagrán & INST & 64 & 50 & 38 & 79,01 & 80,6 & 47,50 \\
\hline
\end{tabular}

Por otro lado, la categoría Twiter (TWIT), obtiene en el GAI una frecuencia del $91,4 \%, N=74$, seguido del GBI $(90,32 \%)$ y $\mathrm{N}=56$ y del GAP con $\mathrm{N}=38$ y un porcentaje del $47,5 \%$, expresándolo como sigue en los diferentes mensajes del foro [GAI_00023](...) Un recurso muy interesante es Twitter (TWIT) para pedirle a mis seguidores opinión o hacer encuestas sobre algunos temas. [GBI_0018](...) Por otro lado tenemos las Redes sociales como Twitter (TWIT), Facebook (FACE), WhatsApp (WHAT), que utilizo para compartir cosas con los demás, como también comunicarme con esas personas, para ello también tenemos Hotmail (OUTL), Gmail (OUTL), Blogger (BLOG), Skype (SKIP), que ayudan a realizar trabajos a distancia. En referencia al uso de Outlook (OUTL), las respuestas obtenidas con mayor puntuación están en el GAI con un porcentaje del $84 \%$ y $\mathrm{N}=68$, seguido del $\mathrm{GBI}$ con un $80,65 \%, \mathrm{~N}=50$ y del GAP con un porcentaje del $60 \%$ y $\mathrm{N}=48$. En cuanto a las respuestas obtenidas en la categoría 3.7 Facebook (FACE), el porcentaje mayor de respuesta aparece en el GBI con un $88,7 \%, \mathrm{~N}=55$, seguida del GAI con un porcentaje de $86,42 \%$ y $\mathrm{N}=70$ y por último el GAP con un porcentaje de respuesta del $55 \%$ con $\mathrm{N}=44$.

El uso de la categoría Skype (SKYP) obtiene una puntuación de $72,6 \%, \mathrm{~N}=45$ en el GBI seguida del GAI con un porcentaje del $51,85 \%, \mathrm{~N}=42$ y del GAP con un $40 \%$ de respuesta y $\mathrm{N}=32$, presentando el interés de 
su uso en el mensaje del foro en los estudiantes [GBI_00048](...) Para trabajar en grupo es muy útil Skype (SKYP) para cuando no podemos quedar personalmente y Drive para elaborar documentos entre varias personas. [GAI_00013](...) Puesto que realizo trabajos grupales, utilizo Drive (DRIV) y Skype (SKYP), siendo éstas muy útiles cuando las distancias entre compañeras es un hecho. Por último, la categoría Instagran (INST), obtiene igual comportamiento en los tres grupos que la anterior categoría, obteniendo el GBI la puntuación más alta, $80,5 \%, \mathrm{~N}=50$, respecto a los demás grupos.

Los resultados obtenidos a nivel de grupos, y que obtienen las mayores puntuaciones, tal y como puede observarse en la fig. 3, hacen referencia al GBI de Educación Infantil, que obtiene la máxima frecuencia en las categorías (WHAP), (SLID), (DRIV), (FACE), (SKYP) e (INST), alcanzando puntuaciones entre [96,8 y 64,5]. El GAl de Educación Infantil, obtiene las puntuaciones más elevadas en las categorías (YOUT), (TWIT) y (OUTL).

\section{CONCLUSIONES}

Las conclusiones del estudio, nos acercan a las herramientas que componen los PLE de los estudiantes de los grupos de maestro, observándose que giran en torno a las tres dimensiones establecidas en el estudio. En cuanto a la (I) primera dimensión, las herramientas de acceso a la información, las conclusiones sobre el uso que hacen los estudiantes, se refieren a bases de datos, objetos de aprendizaje, repositorios institucionales, portales de información específica, entre otros.

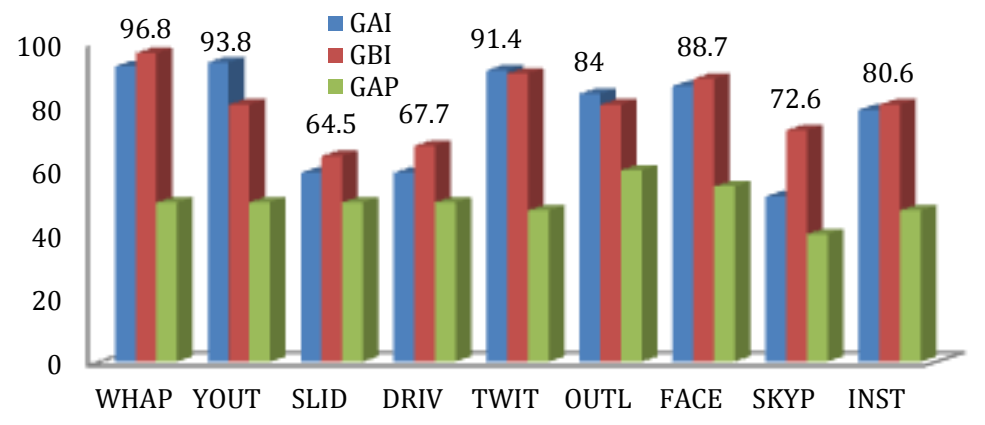

Fig. 3: Dimensión 3. Herramientas de relación con otros

La investigación ha permitido conocer el porcentaje de uso de los recursos tecnológicos empleados por los estudiantes, en base a las categorías establecidas en el estudio para los tres grupos. En referencia a la (II) segunda dimensión, las herramientas de creación y edición de información, las conclusiones nos acercan a un uso de herramientas limitado, centrándose fundamentalmente en el Uso de office, Video, Dibujo y Presentaciones. En cuanto a las conclusiones de la (III) tercera dimensión, las Redes sociales, son consideradas las herramientas de comunicación más fuertes por los estudiantes, destacando Twiter, Facebook y Whatsapp; sobresalen por otro lado, Skype y Youtube. A nivel de los tres grupos del estudio, las conclusiones reflejan un mayor uso de los recursos tecnológicos de los PLE en los estudiantes de los grupos de Educación Infantil respecto de los estudiantes de Educación Primaria, destacando el grupo B de Educación Infantil (GIB).

Por otro lado, en cuanto al uso que le dan a los recursos que componen sus PLE, los estudiantes destacan la realización de actividades de clase, trabajos en grupo, comunicación en general, apreciándose un enfoque pedagógico con una fuerte base tecnológica; se plantea una estrecha relación entre las creencias y expectativas de los estudiantes respecto de las tareas académicas, explicitando el rol de cada herramienta en su proceso de aprendizaje. Se evidencia la coincidencia de determinados recursos tecnológicos en los PLE de todos los estudiantes, así como el uso que le dan en sus prácticas. Por otro lado los resultados nos acercan a la autorregulación y al aprendizaje colaborativo, siendo heterogéneo el nivel de competencia digital de los estudiantes universitarios del estudio.

\section{REFERENCIAS}

Adell, J. y Castañeda, L., Los Entornos Personales de Aprendizaje (PLEs): una nueva manera de entender el aprendizaje. En R. Roig Vila y M. Fiorucci (Eds.). Claves para la investigación en innovación y calidad educativas, Marfil - Roma TRE Universita degli studi, 19-30, Alcoy (2010)

Altman, D.G., Practical statistics for medical research, Chapman and Hall, New York (1991)

Attwell, G., Castañeda, L., y Buchem, I., Invitado prefacio editorial: Edición especial de los entornos personales de aprendizaje, Conferencia de 2011. International Journal of virtuales y entornos de aprendizaje 
personales (IJVPLE), 4 (4), iv-vii (2013)

Attwell, G., Personal Learning Environments: The Future of Education? Presentación visual, disponible en: https://goo.gl/KL8PYv (2008)

Attwell, G., The Personal Learning Environments - the future of eLearning? ISSN 1887-1542, eLearning Papers, 2(1) (2007)

Cabero, J., Barroso, J., y Llorente, M.C., El diseño de Entornos Personales de Aprendizaje y la formación de profesores en TIC, https://goo.gl/EImICx, ISSN 2013-9144, Digital Education Review, 18, 27-37 (2010)

Casquero, O., PLE: Una perspectiva tecnológica. En L. Castañeda y J. Adell (Eds.). Entornos Personales de Aprendizaje: Claves para el ecosistema educativo en red, Alcoy, Marfil, pp. 71-84 (2013)

Castañeda, L. y Adell, J., Beyond the tools: analysing personal and group learning environments in a university course / Más allá de la tecnología: análisis de los entornos de aprendizaje personales y grupales de estudiantes en una asignatura universitaria, DOI: 10.1080/11356405.2014.985946, Cultura y Educación, 26, 4, 739-774 (2014)

Castañeda, L. y Gutiérrez, I., Redes sociales y otros tejidos online para conectar personas. En Castañeda, L. (Coord.). Aprendizaje en redes Sociales. Tejidos educativos en los nuevos entornos. MAD Eduforma, Sevilla (2010)

Castañeda, L. y Soto, J., Patchworking web 2.0 for learning: Introducing ICT as a professional tool building personal learning environments. Proceedings of the The PLE Conference 2010, 8 - 9 July 2010, Barcelona https://goo.gl/CDYpBO (2010)

Cerda, A., Salcedo, A., Pérez, y Marín, V., Futuros Profesores de Matemáticas: Rol de la Disponibilidad Léxica, Esquemas de Razonamiento Formal en Logros Académicos Durante su Formación Inicial. https://dx.doi.org/10.4067/S0718-50062017000100005, Formación Universitaria, 10(1), 33-46 (2017)

Coll, C., Engel, A., Saz, A. y Bustos, A., Personal learning environments: design and use / Los entornos personales de aprendizaje en la educación superior: del diseño al uso, DOI:10.1080/11356405.2014.985935, Cultura y Educación, 26, 4, 775-801 (2014)

Couros, A., Why networked learning matters. Presented at education in a changing environement (ECE) $6^{\text {th }}$ International Conference, Creativity and Engagement in Higher Education, University of Salford, Greater Manchester, U.K., 6-8 July (2011)

Chaves, E., Trujillo, J.M. y López, J.A., Acciones para la autorregulación del aprendizaje en entornos personales. Pixel-Bit. Revista de medios y educación, 48, 67-82 (2016)

Chaves, E., Trujillo, J.M. y López, J.A., Autorregulación del Aprendizaje en Entornos Personales de Aprendizaje en El Grado de Educación Primaria de la Universidad de Granada, España, http://dx.doi.org/10.4067/S0718-50062015000400008, Formación Universitaria, 8 (4), 63-76 (2015)

Drownes, S., Learning networks in practice. Emerging Technologies for Learning, https://goo.gl/bpzGbr, 2, 19-27 (2007)

Feliz, T., Análisis de contenido de la comunicación asíncrona en la formación universitaria. DOI: 104438/1988-592X-RE-2010-358-079, Revista de Educación, 358, 282-309 (2012)

Fiedler, S., y Pata, K., Distributed learning environments and social software: in search for a framework of design. In S. Hatzipanagos y S. Warburton (Eds.). Social software y developing community ontologies, 145158, Hershey, PA, IGI Global. G. (2009)

Fleiss, J.L., Statistical methods for rates and proportions, John Wiley and Sons, New York (1981)

Huber, G., Introducción al análisis de datos cualitativos. En A. Medina y S. Castillo (Coords.) Metodología para la realización de proyectos de investigación y tesis doctorales, 91-129, UNED, Madrid (2003)

Llorente, M.C., Aprendizaje autorregulado y PLE. EDMETIC, Revista de Educación Mediática y TIC, 2(1), 
58-75, https://goo.gl/uizpiv (2013)

Marín-Díaz, V., Sampedro-Requena, B.E. y Vega-Gea, E., Construcción de una escala para determinar la utilidad de los Blogs en la educación superior, Psychology, Society, \& Education, 8(3), 217-228 (2016)

Van Harmelen, M., Design trajectories: four experiments in PLE implementation, DOI: 10.1080/10494820701772686, Interactive Learning Environments, 16, 35-46 (2008)

Valtonen, T., Hacklin, S., Dillon, P., Vesisenaho, M., Kukonen, J. y Hietanene, A., Perspectives on personal learning environments held by vocational students, Computers \& Education, 58, 732-739 (2012) 